Science. Held in Jerusalem, August 26 - September 2, 1964, North-Holland, Amsterdam, pp. $166-178$

[14] W. Szmielew, Some metamathematical problems concerning elementary hyperbolic geometry, The axiomatic method with special reference to geometry and physics, Proceedings of an The axiomatic methos wion held at the University of California, Berkeley, December 26, 1957 International Symposium held at the University of California, Berke

[15] A. Tarski, A decision method for elementary algebra and geometry, Second edition, revised, University of California Press Berkeley and Los Angeles, 1951.

[16] - What is elementary geometry? The axiomatic method with special reference to geometry and physics, Proceedings of an International Symposium held at the University of California, and phy

[17] A. Tarski, A. Mostowski and R. M. Robinson, Undecidable theories, North-Folland, Amsterdam 1953.

[18] O. Veblen, A system of axioms for geometry, Trans. Amer. Math. Soc. 5, 1904, pp. 343-384

[19] A. N. Whitehead, The axioms of descriptive geometry, Cambridge, 1907.

Accepté par la Rédaction le 12. 10. 1976

\section{Non-axiomatizability of real general affine geometry}

by

Alexander Prestel (Konstanz) and Lesław W. Szczerba (Warszawa)

Abstract. In Szczerba-Tarski [7] the question has been reised whether general affine geometry is an elementary theory of affine plane over field of real numbers restricted to non-empty, open and convex set. It is proved that the answer is negative because the first theory is given axiomatically while the second is not recursively axiomatizable.

There is a well known axiom-system of the arithmetic of real numbers containing just one non-elementary axiom, the so called continuity axiom. It follows from the considerations in Tarski [9] (see also Tarski, Mc Kinsey [11]) that by replacing the continuity axiom by the set of all of its first order instances we get an axiom system of the elementary arithmetic of real numbers. The same procedure may be carried out for real Euclidean (see Tarski [10]), hyperbolic and absolute geometry (see Szmielew [8]). In Szczerba-Tarski [6], the question was asked whether the same procedure used in the case of general affine geometry provides an axiom system for elementary real general affine geometry.

We shall prove that it is not true. In fact, elementary real general affine geometry turns out to be non-axiomatizable. Nevertheless the procedure of replacing the continuity axiom by the continuity schema provides us with an axiomatizable subtheory of elementary real general affine geometry which, in some sense, is as close as possible to it. Namely the simplest sentence discriminating these theories is of type $\forall \exists \forall$. The method of proof may be applied to the proof of some other non-axiomatizability results, e.g. we may prove by its means the non-axiomatizability of the elementary theory of rational function fields over formaly real fields, and the already known (see Rautenberg [4]) non-axiomatizability of the elementary theory of Archimedean ordered fields.

§ 1. Theorems on non-axiomatizability. Let $\mathscr{M}$ be a class of structures of the some signature (which will be regarded as fixed for the whole of this section). Let $\mathbf{T}(\mathscr{A})$ be the elementary theory of the class $\mathscr{M}$. 
Assumption. There are formulas

$$
\begin{aligned}
& \varphi\left(v_{0}, \ldots, v_{r}\right), \\
& \varphi_{0}\left(v_{0}, \ldots, v_{r}\right), \\
& \varphi_{1}\left(v_{0}, \ldots, v_{r}\right), \\
& \varphi_{+}\left(v_{0}, \ldots, v_{r}, v_{r+1}, v_{r+2}\right), \\
& \varphi .\left(v_{0}, \ldots, v_{r}, v_{r+1}, v_{r+2}\right), \\
& \psi\left(v_{0}, \ldots, v_{r-1}\right)
\end{aligned}
$$

with the following properties:

(1) There is a structure $\mathfrak{M} \in \mathscr{M}$ and elements $a_{0}, \ldots, a_{r-1}$ of $\left.\mathfrak{l}\right)$ such that

$$
\mathfrak{M} \vDash \psi\left[\begin{array}{lll}
\boldsymbol{v}_{0} & \ldots & \boldsymbol{v}_{r-1} \\
a_{0} & \ldots & a_{r-1}
\end{array}\right] \text {. }
$$

(2) For any $\mathfrak{M} \in \mathscr{M}$ and any sequence $a=\left\langle a_{0}, \ldots, a_{r-1}\right\rangle$ of elements of $\mathfrak{M}$, if

$$
\mathfrak{M} \vDash \psi\left[\begin{array}{lll}
\boldsymbol{v}_{0} & \ldots & \boldsymbol{v}_{r-1} \\
\boldsymbol{a}_{0} & \ldots & \boldsymbol{a}_{r-1}
\end{array}\right]
$$

then

$$
\left\langle\varphi^{\mathfrak{W}, a}, \varphi_{0}^{\mathfrak{2}, a}, \varphi_{1}^{\mathfrak{2}, a}, \varphi_{+}^{\mathfrak{Q}, a}, \varphi_{.}^{\mathfrak{2}, a}\right\rangle \cong\langle\omega, 0,1,+, \cdot\rangle
$$

where $\varphi^{\mathfrak{Q B}, a}, \varphi_{0}^{\mathfrak{M}, a}, \varphi_{1}^{\mathfrak{M}, a}, \ldots, \varphi_{.}^{\mathfrak{M}, a}$ are the sets, elements and operations defined in $\mathscr{M}$ by the formulas $\varphi, \varphi_{0}, \ldots, \varphi$. with the sequence $a$ used as parameters.

In other words, the formulas $\varphi_{0}, \varphi_{1}, \varphi_{+}, \varphi$. define uniformly (with parameters) the arithmetic of natural numbers in the structure of some non-empty subclass $\mathcal{N}$ of the class $\mathscr{M}$. The class $\mathscr{N}$ and admissible parameters are singled out by formula $\psi$. The existence of the formula $\psi$ is essential for our purpose. Without it we would only get undecidability of $\mathbf{T}(\mathscr{M})$ (cf. Tarski-Mostowski-Robinson [12]). If Assumption holds, we say that the arithmetic is interpretable in $\mathbf{T}(\mathscr{M})$.

By the elementary arithmetic of natural numbers we mean

$$
\operatorname{Ar}=\mathbf{T}(\langle\omega, 0,1,+, \cdot,\rangle) .
$$

Let $\alpha$ be any formula of $\mathbf{A r}$ in which + and are treated as ternary relation symbols, but not operation symbols, and 0,1 as unary relation symbols. Then by $\alpha^{\varphi}$ we mean the formula obtained from $\alpha$ by restricting all quantifiers to the formula $\varphi$, and moreover by replacing to relation symbols $0,1,+$ and $\cdot$ by the formulas $\varphi_{0}, \varphi_{1}, \varphi_{+}$. and $\varphi$., respectively.

LEMMA 1.1. If the arithmetic is interpretable in $\mathbf{T}(\mathscr{A})$ and $\alpha$ is an arithmetical sentence, then

$$
\alpha \in \mathbf{A r} \quad \text { iff } \quad\left[\forall v_{0}, \ldots, v_{r-1}\left(\psi \rightarrow \alpha^{\varphi}\right)\right] \in \mathrm{T}(\mathscr{A}) .
$$

Proof. Let $\alpha$ be a sentence true in the arithmetic of natural numbers, i. e. $\alpha \in \mathbf{A r}$. Then for any $\mathfrak{M} \in \mathscr{M}$ and any sequence $\left\langle a_{0}, \ldots, a_{r-1}\right\rangle$ of elements of $\mathfrak{M}$ by part (2) of Assumption we have

$$
\mathfrak{M i} \vDash\left(\psi \rightarrow \alpha^{\varphi}\right)\left[\begin{array}{l}
v_{0}, \ldots, v_{r-1} \\
a_{0}, \ldots, a_{r-1}
\end{array}\right] .
$$

Therefore

$$
\left[\forall v_{0}, \ldots, v_{r-1}\left(\psi \rightarrow \alpha^{\varphi}\right)\right] \in \mathbf{T}(\mathscr{M}) .
$$

If $\alpha \notin \mathbf{A r}$ then $\neg \alpha \in \mathbf{A r}$ and we have

$$
\left[\forall v_{0}, \ldots, v_{r-1}\left(\psi \rightarrow(\neg \alpha)^{\varphi}\right)\right] \in \mathbf{T}(\mathscr{M}) .
$$

But then by part (1) of Assumption

$$
\left[\forall v_{0}, \ldots, v_{r-1}\left(\psi \rightarrow \alpha^{i p}\right)\right] \notin \mathbf{T}(\mathscr{M}) .
$$

This completes the proof of the lemma.

THEOREM 1.2. If arithmetic is interpretable in $\mathbf{T}(\mathscr{A l})$ then $\mathbf{T}(\mathscr{A})$ is not axiomatizable.

Proof. This follows from Lemma 1.1 since Ar is not axiomatizable (see e.g. Tarski-Mostowski-Robinson [12]).

Suppose $\mathbf{T}^{\prime}$ is an axiomatizable subtheory of $\mathbf{T}(\mathscr{N})$ where $\mathscr{M}$ satisfies Assumption. Then there has to be a sentence true in all structures of $\mathscr{U}$ but not belonging to $\mathbf{T}^{\prime}$.

THEOREM 1.3. If the arithmetic is interpretable in $\mathbf{T}(\mathscr{M})$, then there is universal arithmetic formula $\alpha$ with a free variable $x$ such that for any recursively enumerable subset $\mathbf{T}^{\prime}$ of $\mathbf{T}(\mathscr{M})$ (e.g. any axiomatizable subtheory of it) there is a natural number $\boldsymbol{m}_{\mathbf{T}}$ such that

$$
\left[\forall v_{0}, \ldots, v_{r-1}\left(\psi \rightarrow\left[\alpha\left(m_{\mathbf{T}^{\prime}}\right)^{\alpha}\right)\right]\right.
$$

holds in all $\mathfrak{M} \in \mathscr{M}$ but does not belong to $\mathrm{T}^{\prime}$.

Proof. Matiasevich's result (see Matiasevich [3] or Matiasevich [2]) implies, that there is a polynomial $P\left(x_{0}, \ldots, x_{k}\right)$ with the following property: For any recursively enumerable set $A$ of natural numbers there is a number $m$ such that

$$
A=\left\{n:\left[\exists x_{2} \ldots x_{k}\left(P\left(n, m, x_{2}, \ldots, x_{k}\right)=0\right)\right] \in \mathrm{Ar}\right\} .
$$

Let $\mathbf{T}^{\prime}$ be a recursively enumerable subset of $\mathbf{T}(\mathscr{M})$; then

$$
A \boldsymbol{T}^{\prime}=\left\{n:\left[\exists v_{0} \ldots v_{r-1}\left(\psi \rightarrow\left(\neg \exists x_{2} \ldots x_{k} P\left(n, m, x_{2}, \ldots, x_{k}\right)=0\right)^{p}\right)\right] \in \mathbf{T}^{\prime}\right\}
$$

is a recursively enumerable set. Thus there is a number $m_{\mathbf{T}^{\prime}}$ such that

$$
A \boldsymbol{\top}^{\prime}=\left\{n:\left[\exists x_{2} \ldots x_{k} P\left(n, m \mathbf{T}^{\prime}, x_{2}, \ldots, x_{k}\right)=0\right] \in A \mathbf{r}\right\}
$$

For $n=m_{\mathrm{T}^{\prime}}$ we get

$(\stackrel{*}{*}) \quad\left[\exists x_{2} \ldots x_{k} P\left(m \mathrm{~T}^{\prime}, m_{\mathrm{T}^{\prime}}, x_{2}, \ldots, x_{k}\right)=0\right] \in \mathrm{Ar}$

$$
\text { iff }\left[\forall v_{0} \ldots v_{1-1}\left(\psi \rightarrow\left(\neg \exists x_{2} \ldots x_{k} P\left(m_{\mathrm{T}^{\prime}}, m_{\mathrm{T}^{\prime}}, x_{2}, \ldots, x_{k}\right)=0\right)^{\varphi}\right)\right] \in \mathrm{T}^{\prime} .
$$

We put

$$
\alpha(x):=\neg \exists x_{2} \ldots x_{k} P\left(x, x, x_{2}, \ldots, x_{k}\right)=\mathbf{0} .
$$


Suppose $f^{*} \alpha\left(\boldsymbol{m}_{\boldsymbol{T}}\right) \in$ Ar. Then by $\left(\begin{array}{c}* \\ *\end{array}\right)$ we have

$$
\left[\forall v_{0} \ldots v_{r-1}\left(\psi \rightarrow\left[\alpha\left(m_{\mathbf{T}}\right)\right]^{\varphi}\right)\right] \in \mathbf{T}^{\prime} \subseteq \mathbf{T}(\mathscr{M}) \text {. }
$$

But, on the other hand, from Lemma 1.1 we get

$$
\cdot\left[\forall v_{0} \ldots v_{r-1}\left(\psi \rightarrow\left[\neg \alpha\left(\boldsymbol{m}_{\mathbf{T}^{\prime}}\right)\right]^{\varphi}\right)\right] \in \mathbf{T}(\mathscr{M}) \text {. }
$$

Therefore $\neg \alpha\left(\boldsymbol{m}_{\mathbf{T}}\right) \notin \mathbf{A r}$ or, what ammounts to the same, $\alpha\left(\boldsymbol{m}_{\mathbf{T}}\right) \in \mathbf{A r}$. Thus, by $\left(\begin{array}{l}* \\ *\end{array}\right), \forall v_{0} \ldots v_{r-1}\left(\psi \rightarrow\left[\alpha\left(m_{\mathbf{T}^{\prime}}\right)\right]^{\varphi}\right)$ does not belong to $\mathbf{T}^{\prime}$. Nevertheles by Lemma 1.1 it belongs to $\mathbf{T}(\mathscr{M})$. This completes the proof.

Let $\forall_{n}$ be the set of all sentences in prenex normal form having $n-1$ changes of quantifiers, starting with the universal one.

Analogously we define $\exists_{n}$ as the set of all sentences in prenex normal form having $n-1$ changes of quantifiers starting with existential one, since the type of the prenex normal form of the sentence $(*)$ in Theorem 1.3 does not depend on the set $\mathbf{T}^{\prime}$, we get, as a simple consequence, the following

COROLLARY 1.4. If arithmetic is interpretable in $\mathbf{T}(\mathscr{M})$, then there is a natural number $m$ such that no subset $\mathbf{T}^{\prime}$ from $\mathbf{T}(\mathscr{M})$ containing $\mathbf{T}(\mathscr{M}) \cap \forall_{m}$ is recursively enumerable. In particular, there is no axiomatizable subtheory of $\mathbf{T}(\mathscr{M})$ containing the above intersection.

§ 2. Examples of non-axiomatizable algebraic theories. We shall now give some applications of the theorems from Section 1. We begin with a trivial one:

EXAMPle 2.1. Arithmetic of natural numbers. Let $\mathscr{M}$ contain just one structure $\langle\omega, 0,1,+, \cdot\rangle$. By Theorem $1: 2, \mathbf{T}(\mathscr{M})$ is not axiomatizable, and by Theorem 1.3 for any $n \geqslant 1$, the set $\operatorname{Ar} \cap \forall_{n}$ is not recursively enumerable. Both results are well known. In fact, we have used the non-axiomatizability of $\mathbf{T}(\mathscr{M})$ in the proof Theorem 1.2.

EXAMPLe 2.2. Archimedean ordered fields. Let $\mathscr{M}$ be the class of all Archimedean ordered fields. To prove that arithmetic is interpretable in the theory of Archimedean ordered fields we use the formula

$$
\begin{aligned}
& \varrho(n):=\forall a b\left\{\exists x y z x^{2}+a y^{2}=b z^{2}+2 \wedge \forall m\left[\exists x y z x^{2}+a y^{2}=b z^{2}+a b m^{2}+2\right.\right. \\
& \left.\left.\rightarrow \exists x y z x^{2}+a y^{2}=b z^{2}+a b(m+1)^{2}+2\right] \rightarrow \exists x y z x^{2}+a y^{2}=b z^{2}+a b n^{2}+2\right\},
\end{aligned}
$$

stated in Robinson [5] which defines the set of integers in the field $\mathbb{Q}$ of rational numbers, and the sentence

$$
\begin{array}{r}
\varrho(0) \wedge \varrho(1) \wedge \forall x[0<x<1 \rightarrow \neg \varrho(x)] \wedge \forall x y[\varrho(x) \wedge \varrho(y) \rightarrow \varrho(x-y) \wedge \varrho(x \cdot y)] \wedge \\
\wedge \forall x \exists y z[\varrho(y) \wedge \varrho(z) \wedge x \cdot y=z]
\end{array}
$$

(from Rautenberg [4]), which is true in an Archimedean ordered field $\tilde{f}$ only if it is the field of rational numbers. By Theorem 1.2 we get the non-axiomatizability of $\mathbf{T}(\mathscr{M})$, proved already in Rautenberg [4]. Let us consider a theory $\mathbf{T}^{\prime}$ based upon axioms obtained from those of the non-elementary theory of Archimedean ordered fields by replacing the only non-elementary Archimedean axiom by its first order instances. The formula from Theorem 1.3 provides a solution of Problem 3 in Rautenberg [4]. By Corollary 1.4 there is a natural number $m$ such that the set $\mathbf{T}(\mathscr{M}) \cap \forall_{m}$ is not contained in any recursively enumerable subset of $\mathbf{T}(\mathscr{M})$. We may calculate the number $m$ from the formulas $\varphi$ and $\psi$. We then obtain $m=6$, though the least $m=2$. In fact, $T(\mathscr{M}) \cap \forall_{1}$ is itself a recursively enumerable set. This follows from

THEOREM 2.2.1. Let $\mathbf{T}^{\prime}$ be the theory of ordered fields. Then

(i) $\mathbf{T}(\mathscr{M}) \cap \forall_{1}=\mathbf{T}^{\prime} \cap \forall_{1}$ and $\mathbf{T}(\mathscr{M}) \cap \exists_{1}{ }^{\prime}=\mathbf{T}^{\prime} \cap \exists_{1}$,

(ii) the sentence

$\left(\begin{array}{c}* \\ * * *\end{array}\right)$

$$
\forall x \exists y\left(0<x \rightarrow x<y^{2} \wedge y^{2}<x+1\right)
$$

belongs to $\mathbf{T}(\mathscr{M})$ but not to $\mathbf{T}^{\prime}$.

Proof. (i) Since $\mathbf{T}^{\prime} \subseteq \mathbf{T}(\mathscr{M})$, the inclusions

$$
\mathbf{T}^{\prime} \cap \forall_{1} \subseteq \mathbf{T}(\mathscr{M}) \cap \forall_{1} \quad \text { and } \quad \mathbf{T}^{\prime} \cap \exists_{1} \subseteq \mathbf{T}(\mathscr{M}) \cap \exists_{1}
$$

are obvious. Any ordered field may be extended to a real closed ordered field which is elementarily equivalent to the field of real numbers which is Archimedean-ordered. Thus

$$
\mathbf{T}(\mathscr{M}) \cap \forall_{1} \subseteq \mathbf{T}^{\prime} \cap \forall_{1} .
$$

On the other hand, the field of rational numbers, which is also Archimedean-ordered, may be embedded in any ordered field and therefore

$$
\mathbf{T}(\mathscr{M}) \cap \exists_{1} \subseteq \mathbf{T}^{\prime} \cap \exists_{1} \text {. }
$$

(ii) The sentence $\left(\begin{array}{c}* \\ * *\end{array}\right)$ holds in all Archimedean-ordered fields since the set of rational numbers is dense in any of them. It is easy to prove that the sentence $(\underset{* *}{*})$ does not hold in a field of rational functions in one variable $t$ over the field of reals for the ordering in which $t$ is greater than any real number. In fact, there is no square between $t$ and $t+1$.

EXAMPLE 2.3. Rational function fields in one variable over formally real fields. Let $\mathscr{M}$ be the class of all rational function fields in one variable over formally real fields, i.e., fields in which -1 is not a sum of squares. It is easy to see that $\mathbf{T}(\mathscr{M})$ is the theory of rational functions (of at least one variable) over formally real fields. Any field from the class $\mathscr{M}$ is formally real. Thus the theory $\mathbf{T}^{\prime}$ of formally real fields is a subtheory of $\mathbf{T}(\mathscr{H})$. To prove that the arithmetic is interpretable in $\mathbf{T}(\mathscr{M})$ we let $\varphi$ be the formula Nat from [5] p. 309. Using formula Con from [5] p. 308 we set

$$
\psi:=\forall x\left(\operatorname{Con} x \rightarrow \operatorname{Con}(x+1) \wedge \forall n v w \exists x y n^{2}+v^{2}+w^{2}=x^{2}+y^{2}\right) .
$$

As in [5] we prove that $\tilde{F} \vDash \psi$ implies that Nat defines set of natural numbers in $\mathfrak{F} \in \mathscr{H}$.

THEOREM 2.3.1. (i) $\mathbf{T}(\mathscr{A}) \cap \forall_{2}=\mathbf{T}^{\prime} \cap \forall_{2}$.

(ii) The sentence $\exists \boldsymbol{x} \forall \boldsymbol{y} \neg \mathbf{1}+\boldsymbol{x}^{2}=y^{2}$ belongs to $\mathbf{T}(\mathscr{M})$ but not to $\mathbf{T}^{\prime}$. 
Proof of (i). Since $\mathbf{T}^{\prime} \subseteq \mathbf{T}(\mathscr{M})$, it will suffice to prove $\mathbf{T}(\mathscr{M}) \cap \forall_{2} \subseteq \mathbf{T}^{\prime}$. Let $\alpha \in \mathbf{T}(\mathscr{M}) \cap \forall_{2}$ and let $\mathfrak{F}$ be any formally real field. By the Lowenheim-SkolemTarski theorem, there is a countable field $\widetilde{F}^{\prime}$ elementarily equivalent to $\widetilde{F}$. Thus $\widetilde{F}^{\prime}$ is a formally real field. Hence it contains (up to an isomorphism) the field $\mathfrak{Q}$ of rational numbers. We may assume that $\mathfrak{F}^{\prime}$ has countable transcendence degree over $\mathfrak{Q}$. We shall prove that $\widetilde{F}^{\prime} \vDash \alpha$ and therefore $\widetilde{F} \vDash \alpha$.

Let $b$ be a sequence of elements of $\widetilde{F}^{\prime}$ such that $b_{n}$ is transcendent over $\widetilde{F}_{n}$ and such that $\mathfrak{F}=\bigcup_{n \in \omega} \mathfrak{F}_{n}\left(b_{n}\right)$. Since $\mathbb{F}_{n}=\mathfrak{F}_{n-1}\left(b_{n-1}\right) \in \mathscr{M}$, by assumption $\mathbb{F}_{n} \vDash \alpha$. Now, $\widetilde{\mho}^{\prime}=\bigcup_{n \in \omega} \widetilde{\mho}_{n} \vDash \alpha$ follows from $\alpha \in \forall_{2}$ by the Loś-Suszko theorem (see Loś -Suszko [1]).

(ii) Since the rational function $1+t^{2}$ is not a square of any rational function, we have

$$
\left(\exists x \forall y \neg \mathbf{1}+x^{2}=y^{2}\right) \in \mathrm{T}(\mathscr{A}) .
$$

On the other hand, this sentence is false in the field of real numbers, and therefore

$$
\left.(\exists x \forall y\urcorner \mathbf{1}+x^{2}=y^{2}\right) \notin \mathrm{T}^{\prime} .
$$

EXAMPLE 2.4. General affine geometry. Let $\mathfrak{S}_{2}(\mathfrak{R})$ be the affine plane over the field of real numbers. A restricted affine plane (a restricted real affine plane) is a restriction of $\mathfrak{Q}_{2}(\mathfrak{R})$ to any subset $S$ of $R^{2}$. (For exact definitions as well as for the meaning of the notions used in the subsequent text see Szczerba-Tarski [7].) Let $\mathscr{A}$ be the class of all restricted Cartesian planes $\mathfrak{2}_{2}(\mathfrak{R}, S)$ where $S$ is a non-empty, open convex set in $\mathfrak{A}_{2}(\mathfrak{R})$. In Szczerba-Tarski [7] the axiomatizable theory $\mathbf{G A}_{2}$, called general affine (plane) geometry, is defined. It is proved that $\mathbf{T}(\mathscr{A})$ is an extension of $\mathbf{G A}_{2}$, i.e., if $\mathfrak{Q} \in \mathscr{A}$ then $\mathfrak{Q}=\mathbf{G A}_{2}$. For the sake of simplicity we shall consider a class $\mathscr{A}^{\prime} \subseteq \mathscr{A}$ of those restricted real Cartesian planes from $\mathscr{A}$ which contain the points $e_{0}=(0,0), e_{1}=\left(\frac{1}{2}, 0\right), \epsilon_{1}^{\prime}=\left(\frac{1}{2},-\frac{1}{2}\right), e_{\infty}=(1,0)$ and $e_{\omega}=(0,1)$. It is easy to see that for any structure $\mathfrak{Q} \in \mathscr{A}$ there is an isomorphic structure $\mathfrak{Q}^{\prime} \in \mathscr{A}^{\prime}$. Let $\mathscr{A}^{+}$ be the class of all expansions of structures of $\mathscr{A}$ by points $e_{0}, e_{1}, e_{1}^{\prime}, e_{\infty}$ and $e_{10}$, i.e., structures of the form $\left\langle A, e_{0}, e_{1}, e_{1}^{\prime}, \epsilon_{\infty}, e_{\omega}, B\right\rangle$ where $\langle A, B\rangle \in \mathscr{d ^ { \prime }}$.

THEOREM 2.4.1. The arithmetic is interpretable in $\mathrm{T}\left(\mathscr{A}^{+}\right)$.

Proof. To prove the theorem we have to provide an interpretation of the arithmetic of natural numbers in a definable subclass of $\mathscr{A}^{+}$. To do this we shall need functions $\mathbf{H}^{+}$and $\mathbf{H F}^{+}$mapping the language $\mathbf{L}(\mathbf{0}, \mathbf{1},+, \cdot, \leqslant)$ into $\mathbf{L}(\boldsymbol{B})$ and formula $\boldsymbol{K}^{+} \in \mathbf{L}(\boldsymbol{B})$ meaning: "the given two numbers are coordinates of a given point". We shall define them by means of functions $\mathbf{H}, \mathbf{H F}$ and formula $\boldsymbol{K}_{5}$ defined in Szczerba-Tarski [7] (see Definitions 2.13 and 2.16, and Section 5). The definitions are as follows:

$$
\mathbf{H}^{+}(\alpha)=\mathbf{H}(\alpha)\left(\begin{array}{llll}
v_{0} & v_{1} & v_{2} & v_{3} \\
e_{\omega} & e_{1} & e_{\infty} & e_{0}
\end{array}\right)
$$

$$
\begin{gathered}
\mathbf{H F}^{+}(\alpha)=\mathbf{H F}(\alpha)\left(\begin{array}{llll}
v_{0} & v_{1} & v_{2} & v_{3} \\
e_{0} & e_{1} & e_{\infty} & e_{00}
\end{array}\right), \\
K^{+}=K_{5}\left(\begin{array}{lllll}
v_{0} & v_{1} & v_{2} & v_{3} & v_{4} \\
e_{0} & e_{1} & e_{\infty} & e_{00} & e_{1}^{\prime}
\end{array}\right) .
\end{gathered}
$$

To define the arithmetic of natural numbers we use the following formulas:

$$
\begin{aligned}
& \varphi=B\left(e_{0} v_{0} e_{\alpha_{\alpha}}\right) \wedge v_{0} \neq e_{\alpha_{1}} \wedge \\
& \wedge \forall v_{8} v_{9} v_{10} v_{11} v_{12}\left[B\left(e_{0} v_{0} v_{12}\right) \wedge K^{+} \rightarrow \mathrm{HF}^{+}\left(v_{0}^{2}+v_{1}^{2} \leqslant 4\right)\right], \\
& \varphi_{0}=\left(v_{0}=e_{0}\right), \\
& \varphi_{1}=\left(v_{0}=e_{1}\right) \text {, } \\
& \varphi_{++}=\boldsymbol{H}^{+}\left(v_{0}+v_{1}=v_{2}\right)\left(\begin{array}{lll}
v_{8} & v_{9} & v_{10} \\
v_{0} & v_{1} & v_{2}
\end{array}\right), \\
& \varphi .=H^{+}\left(v_{0}+v_{1}=v_{2}\right)\left(\begin{array}{lll}
v_{8} & v_{9} & v_{10} \\
v_{0} & v_{1} & v_{2}
\end{array}\right), \\
& \psi=\varphi\left(e_{0}\right) \wedge\left[\forall v_{0} v_{1} \varphi \wedge \varphi_{+}\left(v_{0}, e_{1}, v_{1}\right) \rightarrow \varphi\left(v_{1}\right)\right] \wedge \\
& \wedge\left[\forall v_{0} v_{1} v_{2} \varphi \wedge \varphi_{+}\left(v_{0}, e_{1}, v_{2}\right) \wedge B\left(v_{0} v_{1} v_{2}\right) \wedge \varphi\left(v_{1}\right)\right. \\
& \left.\rightarrow v_{0}=v_{1} \vee v_{1}=v_{2}\right] \text {. }
\end{aligned}
$$

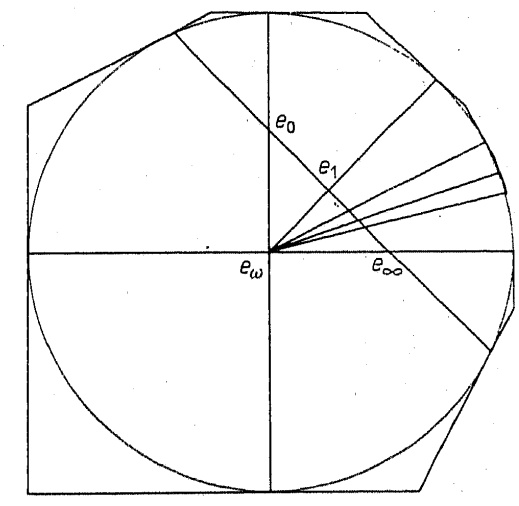

The intuitive meaning of this formulas is easy to see in Fig. 1. The point $a$ is a natural number (i.e. satisfies the formula $\varphi$ ) if it belongs to the segment $\left\langle e_{0} e_{\omega}\right.$ ) and the length of the ray from $e_{\infty}$ through $a$ in $\mathfrak{Q t}$ is equal to 2 . In the case of the formula $\psi$ it is said that

(i) zero (i.e. the point $e_{0}$ ) is a natural number,

(ii) if $a$ is a natural number, then $a+1$ is a natural number, 
(iii) there are no natural numbers between $a$ and $a+1$ exept these two numbers themselves.

Thus in any model of $\psi$ belonging to $\mathscr{A}$, the formulas $\varphi, \varphi_{0}, \varphi_{1}, \varphi_{+}, \varphi$. define the arithmetic of natural numbers. The sets

$$
P_{n}=\left\{\left(x_{1}, x_{2}\right):-2 \sqrt{n^{2}+1}+x_{1}+n x_{2}<0\right\},
$$

are the halfplanes determined by the lines $-2 \sqrt{n^{2}+1}+x_{1}+n x_{2}=0$ containing the point $(0,0)$. Let $S=\bigcap P_{n}$ (see Fig. 1). It is not difficult to see that $\mathfrak{T}_{2}(\mathfrak{R}, S)$ is a model of $\psi$. This completes the proof.

From Theorem 2.4.1 we get

COROLlary 2.4.2. The arithmetic is interpretable in $\mathbf{T}\left(\mathscr{A}^{\prime}\right)$. and therefore

Corollary 2.4.3. The theories $\mathbf{T}\left(\mathscr{A}^{\prime}\right)$ and $\mathbf{T}\left(\mathscr{A}^{+}\right)$are not axiomatizable.

By Theorem 1.3 for any recursively enumerable theory $\mathbf{T} \subseteq \mathbf{T}\left(\mathscr{A}^{+}\right)$, in particular $\mathbf{G A}_{2}^{+}$, there is an universal sentence $\alpha$ such that the implication $\psi \rightarrow \alpha^{\varphi p}$ belongs to $\mathbf{T}\left(\mathscr{A}^{+}\right)$but not to $\mathbf{G A}_{2}$. To calculate the type of the sentence $\psi \rightarrow \alpha^{\text {qp }}$ we have to mention that the formulas $\varphi_{+}$and $\varphi$., being existential, may be replaced equivalently by universal ones. Thus we may assume that the formula $\varphi$ is universal and therefore that the type of the sentence $\alpha^{\varphi}$ is $\forall_{2}$. The sentence $\psi$ is $\forall_{2}$, and thus the sentence $\psi \rightarrow \alpha^{\varphi}$ is $\forall_{2}$.

In the case of the recursively enumerable theory $\mathbf{G A}_{2} \subseteq \mathbf{T}\left(\mathscr{\mathcal { L } ^ { \prime }}\right)$ we may proceed similarly except that we have to replace constants $\boldsymbol{e}_{0}, \boldsymbol{e}_{1}, \boldsymbol{e}_{1}^{\prime}, \boldsymbol{e}_{\omega}, \boldsymbol{e}_{\omega}$ by variables bounded by universal quantifiers. Then we get a $\forall_{2}$ sentence belonging to $T\left(\mathscr{A}^{\prime}\right)$ and therefore to $\mathbf{T}(\mathscr{A})$ but not to $\mathbf{G A}_{2}$

We shall prove that $\forall_{2}$ is the lowest type of sentence discriminating between $\mathbf{G A}_{2}$ and $\mathbf{T}(\mathscr{A})$.

THEOREM 2.4.4. (i) There is a sentence $\alpha$ of type $\forall_{3}$ such that $\alpha \in \mathbf{G A}_{2} \backslash \mathbf{T}(\mathscr{A})$.

(ii) $\mathbf{G A}_{2} \cap \exists_{2}=\mathbf{T}(\mathscr{A}) \cap \exists_{2}$.

The proof of the part (i) is given above. In part (ii) the inclusion

$$
\mathbf{G A}_{2} \cap \exists_{2} \subseteq \mathbf{T}(\mathscr{A}) \cap \exists_{2}
$$

is obvious. Let $\alpha \in\left(\mathbf{T}(\mathscr{A}) \cap \exists_{2}\right) \backslash\left(\mathbf{G A}_{2} \cap \exists_{2}\right)$. Thus there is a model $\mathfrak{Q}$ of $\mathbf{G A}_{2}$ and of $7 \alpha$. Since $\mathfrak{A} \vDash \mathbf{G A}_{2}$, there is a real closed field $\mathfrak{F}$ and a set $S$ of points of $\mathfrak{Y I}_{2}(\mathfrak{F})$ such that $\mathfrak{I}_{2}(\mathfrak{F}, S) \cong \mathfrak{A}$. Thus $\mathfrak{H}_{2}(\mathfrak{F}, S) \vDash \neg \alpha$. We may assume, without loss of generality, that $(0,0) \in S$. Let

$$
\mathfrak{U}^{\lambda}=\mathfrak{P}_{2}\left(\mathfrak{F}, S^{\lambda}\right)
$$

where $S^{\lambda}=\{\lambda a: a \in S\}$. Since for $\lambda \neq 0$ we have $\mathfrak{Q}^{\lambda} \cong \mathfrak{H}$, we obtain $\mathfrak{Q P}^{\lambda} \neq 7 \alpha$ or $\lambda=0$. The set of structures $\mathfrak{A}^{\lambda}$ for $\lambda>0$ is directed, and $7 \alpha$ is of type $\forall_{2}$; therefore by the Łoś-Suszko theorem (see $\operatorname{Loś}_{-}$Suszko [1]) $\left.\bigcup_{\lambda>0} \mathfrak{Y}^{\lambda} \vDash\right\urcorner \alpha$. It is easy to see that $\bigcup_{\lambda>0} \mathfrak{H}^{\lambda}=\mathfrak{A}_{2}(\mathbb{F})_{\alpha} \equiv \mathfrak{Q}_{2}(\mathfrak{R})$. Therefore $\mathfrak{R}_{2}(\mathfrak{R}) \vDash \neg \alpha$, contrary to the assumption that $\alpha \in \mathbf{T}(\mathscr{A})$.

Remark. Theorem 2.4.4(i) and Theorem 2.4.3 give an answer to the problems. posed in 3.11 of Szczerba-Tarski [7].

Modyfying the proof of Theorem 2.4.4(ii) we may strengthen it up:

THEOREM 2.4.5. If a sentence $\alpha$ is of the form

(i) $\alpha=\forall \mathbf{v}_{0} \forall \mathbf{v}_{1} \beta$ where $\beta \in \exists_{2}$,

(ii) $\alpha=\forall \mathbf{v}_{0} \forall \mathbf{v}_{1} \forall \mathbf{v}_{2} \gamma$ where $\gamma \in \exists_{1}$

then $\alpha \in \mathbf{G A}_{2}$ if and only if $\alpha \in \mathbf{T}(\mathscr{A})$.

Proof. Since $\mathbf{G A}_{2} \subseteq \mathbf{T}(\mathscr{A})$ the implication to the right is obvious. Suppose then, that there is a model $\mathfrak{Q r}$ of $\mathbf{G A}_{2}$ and of $\neg \alpha$. Since $\mathfrak{A} \vDash \mathbf{G A}_{2}$ therefore there is a real closed field $\mathfrak{F}$ and a set $S$ of points of $\mathfrak{Q}_{2}(\mathfrak{F})$ such that $\mathfrak{A}_{2}(\mathfrak{F}, S) \cong \mathfrak{Q T}$. Part (i) of the proof: The sentence $\neg \alpha$ is of the form $\exists \mathbf{v}_{0} \exists \mathbf{v}_{1} \neg \beta$ and $\neg \beta \in \forall_{2}$. Therefore there are two points $a_{0}, a_{1} \in S$ satisfying the formula $\neg \beta$ in $\mathfrak{R}_{2}(\mathfrak{\Im} ; S)$. Without the loss of generality we may assume that $a_{0}=(0,0)$ and $a_{1}=(1,0)$. Consider a line $L=\{(x, 0): x \in \mathbb{F})$ in $\mathfrak{H}_{2}(\mathfrak{F})$. Since in $\mathbf{G A}_{2}$ we may prove the existence of (hyperbolic) parallel to any line, there are possible three cases: the line $L$ is included in $S, L \cap S$ is a halfline with origin $(\lambda, 0)$ for some $\lambda \in \mathbb{F}$ and finally $L \cap S$ is an open segment with endpoints $(\lambda, 0),(\mu, 0)$ for some $\lambda, \mu \in \mathbb{F}$. We shall assume that the last case holds. The proof in the remaining cases is analogous. Consider now an affine transformation $f_{\tau}$ mapping a point $\left(x_{0}, x_{1}\right)$ onto the point $\left(x_{0}, \tau x_{1}\right)$. Let $A^{\tau}$ be an image of the structure $\mathfrak{P}_{2}(\mathfrak{F}, S)$ with respect to $f_{\tau}$, i.e.

$$
\mathfrak{Q}^{\mathfrak{\tau}}=\mathfrak{Q}_{2}\left(\mathfrak{F},\left\{\left(x_{0}, \tau x_{1}\right):\left(x_{0}, x_{1}\right) \in S\right\}\right) .
$$

It is obvious that if $0<\tau \leqslant \tau^{\prime}$ then $\mathfrak{Q l}^{\tau}$ is a substructure of $\mathfrak{P}^{\mathfrak{\tau}^{\prime}}$, thus by Łoś-Suszko theorem (see $Ł o s-S u s z k o$ [1]) the sentence $7 \alpha$ is true in

$$
\bigcup_{\tau>0} \mathfrak{Q}^{\tau}=\mathfrak{N}_{2}\left(\mathfrak{\mho},\left\{\left(x_{0}, x_{1}\right): \lambda<x_{0}<\mu\right\}\right)
$$

and therefore $\neg \alpha$ is true in a model of $\mathbf{T}(\mathscr{A})$. Contradiction.

Part (ii) of the proof: the sentence $\neg \alpha$ is of the form $\exists \mathbf{v}_{0} \exists \mathbf{v}_{1} \exists \mathbf{v}_{2} \neg \gamma$ and $\neg \gamma \in \forall V_{1}$. There are points $a_{0}, a_{1}, a_{2} \in S$ satisfying $\neg \gamma$ in $\mathfrak{P}_{2}(\mathscr{F}, S)$. It is easy to see that there is an open triangle $T$ containing $a_{0}, a_{1}, a_{2}$ and included in $S$. Since $\mathfrak{P}_{2}(\mathbb{F}, T)$ is a substructure of $\mathfrak{U}_{2}(\tilde{x}, S)$ containing $a_{0}, a_{1}, a_{2}$ and $\neg \gamma \in \forall_{1}$ therefore $\mathfrak{A}_{2}(\mathscr{F}, T) \vDash \neg \alpha$, but $\mathfrak{V}_{2}(\tilde{F}, T) \vDash \mathbf{T}(\mathscr{A})$ and thus there is a model of $\mathbf{T}(\mathscr{A})$ in which $\neg \alpha$ is true. Contradiction.

\section{Reference}

[1] J. Łoś and R. Suszko, On the extending of models (IV), Fund. Math, 44 (1957), pp. 52-60 [2] Ju. V. Matiasevich, The Diophantiness of enumerable sets, Soviet. Math. Dokl. 11 (1970), pp. $354-358$. 
[3] Ju. V. Matiasevich, Diofantovo predstavlene perecislimych predlkatov, Xzv. Akad. Nauk SSSR. Ser. Mat. 35 (1971), pp. 3-30.

[4] W. Rautenberg, Elementare Schemata Nichtelementarer Axiome, Zeit. Math. Logik und Grund. Math., 13 (1967), pp. 329-366.

[5] J. Robinson, The decision problem for fields, The theory of models, North-Holland, Amsterdam 1965, pp. 299-311.

[6] L.W. Szczerba and A. Tarski, Metamathematical properties of some affine geometries, Logic, Methodology and Philosophy of Science, Proceedings the 1964 International Congress, North Holland, Amsterdam 1965, pp. 166-178.

[7] - - Metamathematical discussion of some affine geometries, Fund. Math. 104 (1979), pp. $155-192$.

[8] W. Szmielew, Some metamathematical problenis concerning elementary hyperbolic geometry, The axiomatic methods with special references to geometry and physics, North-Holland, Amsterdam 1959.

[9] A. Tarski, The Completeness of Elementary Algebra and Geometry, Hermann Cie, Paris 1940.

10] - What is elementary geometry? The axiomatic method with special reference to geometry and physics, North-Holland, Amsterdam 1959.

[11] - and J. C. C. Mc Kinsey, A decision method for elementary algebra and geometry U.S. Air Force Project RAND, R-109. The RAND Corporation, Santa Monica 1948.

[12] - A. Mostowski and R. W. Robinson, Undecidable Theories, North Holland, Amsterdam 1953

\section{Uniform approximation by real functions}

\section{Donald E. Marshall and Anihony G. O'Farrell (Maynooth)}

Abstract. Let $C(X)$ denote the space of real-valued continuous functions on a compact Hausdorff space $X$. We obtain a necessary and sufficient condition for the vectorspace sum $A_{1}+A_{2}$ of two subalgebras to be dense in $C(X)$. We solve the analogous problem for finitely-generated modules over a subalgebra of $C(X)$. Also, we determine the conditions under which these various spaces are closed.

In this paper, we consider some questions about approximation by real functions. The Stone-Weierstrass theorem completed the theory of qualitative uniform approximation by elements of an algebra of real functions. We study two related structures, namely modules and finite sums of algebras. The reader will observe that the nature of the subject is essentially geometric, in contrast to the topological and metric character [3], [6] of complex polynomial approximation. Even a simple rotation of a set in $\boldsymbol{R}^{n}$ can radically alter the closure of certain polynomial spaces.

Let us introduce some notation. If $X$ is a compact Hausdorff space, then $C(X)$ denotes the space of all continuous, real-valued functions on $X$. If $A$ is a subset of $C(X)$, then $\bar{A}$ denotes the closure of $A$ with respect to the uniform norm, $\|\cdot\|$, on $X$ If $f_{1}, f_{2}, \ldots, f_{n}$ belong to $C(X)$, then $P\left(f_{1}, f_{2}, \ldots, f_{n}\right)$ denotes the algebra of al polynomials in $f_{1}, f_{2}, \ldots, f_{n}^{\prime}$ with real coefficients.

Our main result is Proposition 2, which gives a necessary and sufficient condition for the sum of two subalgebras of $C(X)$ to be dense in $C(X)$

We are grateful to Robert Green for helpful conversation.

\section{Sums of algebras.}

(1.1) We begin with a special case. For which functions $f$ and $g$, belonging to $C(X)$, is

$$
P(f)+P(g)=C(X) ?
$$

Clearly it is necessary that the map from $X$ to $R^{2}$, given by $F(x)=(f(x), g(x))$, be injective. Suppose $F$ is injective. If $Y$ is the image of $X$ under $F$, then $Y$ is homeomorphic to $X$. A moment's thought reveals that (1) holds if and only if

$$
P(x)+P(y)=C(Y),
$$

